\title{
Characterisation of a Nafion film by optical fibre Fabry-Perot interferometry for humidity sensing
}

\author{
Juliana S. Santos ${ }^{a}$, Ivo M. Raimundo Jr. ${ }^{\mathrm{a}, *}$, Cristiano M.B. Cordeiro $^{\mathrm{b}}$, Claudecir R. Biazoli $^{\mathrm{b}}$, \\ Carlos A.J. Gouveiac ${ }^{c}$, Pedro A.S. Jorge ${ }^{c}$ \\ a Institute of Chemistry, University of Campinas, Unicamp, P.O. Box 6154, 13083-970 Campinas, Brazil \\ b Institute of Physics “Gleb Wataghin”, University of Campinas, Unicamp, Rua Sérgio Buarque de Holanda, 777, Cidade Universitária Zeferino Vaz, \\ 13083-859 Campinas, Brazil \\ ' INESC Porto, Rua do Campo Alegre 687, Porto 4169-007, Portugal
}

\section{A R T I C L E I N F O}

\section{Article history:}

Received 10 September 2013

Received in revised form 3 January 2014

Accepted 28 January 2014

Available online 6 February 2014

\section{Keywords:}

Relative humidity sensor

Interferometry

Fabry-Perot

Nafion

\begin{abstract}
A B S T R A C T
Nafion has been evaluated as a sensing phase of an optical fibre humidity sensor based on a low-finesse Fabry-Perot interferometer. The sensor was constructed by manual deposition of a drop of a Nafion solution on the tip of a single mode optical fibre, forming a Fabry-Perot resonant cavity. The absorption of water by the Nafion film makes it swells, changing its refractive index and the length of the cavity, which produces a phase shift in the interference signal. The sensitivity, stability and response time of the sensor were evaluated in the $\mathrm{RH}$ range from 22 to $80 \%$ by analysing the correspondent reflection spectra of the interference fringes. As a result, it was obtained that Nafion can be used as sensing phase of an optical fibre humidity sensor based on optical fibre Fabry-Perot interferometry, presenting a response time of $242 \mathrm{~ms}$ ( $3 \%$ RH variation) and a sensitivity of $3.5 \mathrm{~nm} / \% \mathrm{RH}$.
\end{abstract}

(c) 2014 Elsevier B.V. All rights reserved.

\section{Introduction}

Relative humidity (RH) is a fundamental physico-chemical parameter in industrial processes and scientific researches. Although its definition is relatively simple, the ratio of the actual water vapour pressure to the saturated vapour pressure at a specific temperature and pressure, the measurement of humidity continues being a challenge once it is extensively sensible to the physical parameters of the environment. As a consequence, this fact still encourages research activities for the development of sensors capable of sensing over a wide humidity range from 0 to $100 \%$.

Studies regarding the development of sensors that responds to a large dynamic range of $\mathrm{RH}$, based on measurements of electrical and optical properties, have been described, such as capacitive, resistive [1,2], piezoelectric [3], chilled mirror hygrometer [4], psychrometer [5] and infrared optical absorption [6] sensors. Beyond these possibilities, optical fibre sensors have proved to be a useful technology in view of their adaptability in several circumstances and high efficiency. Humidity sensing with optical fibre has been accomplished using different transduction principles, such as interferometry [7,8], resonance [9-11], evanescent waves [12,13] and spectrophotometry [14], allowing the

\footnotetext{
* Corresponding author. Tel.: +55 193521 3136; fax: +55 1935213023

E-mail address: ivo@iqm.unicamp.br (I.M. Raimundo Jr.).
}

measurement of the intensity of light and also of other related properties, such as lifetime, refractive index, scattering, diffraction and polarisation [15]. Optical fibre interferometric sensors have been demonstrated to be suitable options for humidity sensing due to their simple configuration, small size, high sensitivity, efficiency and resolution [16]. For instance, Sagnac interferometry has been used for $\mathrm{RH}$ sensing with a sensitivity of $0.081 \mathrm{~nm} / \% \mathrm{RH}$, operating in the range 20-95\%RH [17]. Alternatively, sensors based on Mach-Zehnder interferometry have been proposed, with sensitivity of $-0.047 \mathrm{~nm} / \% \mathrm{RH}$ in the range $50-90 \% \mathrm{RH}$ [18] and $0.0099 \mathrm{~nm} / \% \mathrm{RH}$ in the range $60-100 \% \mathrm{RH}$ [19]. Fabry-Perot interferometers have also been largely employed as optical fibre sensors for RH, due to their high sensitivity, compactness and easy fabrication. By monitoring changes in the optical cavity, it is possible to measure RH with high resolution and accuracy. These probes reached sensitivity as high as $\sim 5.9 \mathrm{~nm} / \% \mathrm{RH}$ in the range from 88 to $98 \% \mathrm{RH}$ [20] and $0.13 \mathrm{~nm} / \% \mathrm{RH}$ in the $20-95 \% \mathrm{RH}$ range [21].

Generally, optical fibre Fabry-Perot interferometric sensors to detect humidity are produced by depositing a hydrophilic coating material on the optical fibre tip, constituting the sensor probe. Due to the low refractive index contrast of the fibre and sensing material, the reflectivity at the interfaces is small, therefore the behaviour of the cavity is that of a low finesse cavity and identical to a two-beam interferometer. In this case, the interference occurs in the volume defined by the film, between the electromagnetic waves reflected at the fibre-film interface and those reflected 
at the film-external medium interface. For this configuration and application, the ability of water absorption by the material plays an essential role on the sensor performance, once the optical signal is modulated according to the refractive indexes and thicknesses modified by the amount of water absorbed. Several polymers have been used for RH sensors fabrication, including PVA [22], agarose [23], chitosan [21] and PEG [24].

Presenting high hydrophilicity, chemical and thermal stability, high conductivity and mechanical toughness, Nafion is a suitable material for many applications. Developed by DuPont, it is a perfluorosulfonate ion exchange polymer wherein hydrophilic perfluorinated ether side chains end with sulphonate groups, which are periodically attached to hydrophobic perfluoroethylene backbone molecules. Nafion films have been extensively used in fuel cell [25] as well as in electrochemical [26] and optical sensors. For humidity sensing, Nafion is usually used as a matrix for casting films doped with different dyes in which $\mathrm{RH}$ is determined according to their spectrophotometric properties [14,27-32].

In this work, Nafion has been studied as a sensing phase of an optical fibre humidity sensor based on a low-finesse Fabry-Perot interferometer, being an alternative to the existing optical sensors. The sensor probe was assembled by coating the tip of a single mode optical fibre with Nafion, whose refractive index and thickness change with humidity due to the absorption of water vapour, inducing, as a consequence, an optical path modulation. The sensitivity, stability and response time of the sensor have been quantified in the $\mathrm{RH}$ range from 22 to $80 \%$ by analysing the correspondent reflection spectra of the interference fringes.

\section{Sensing principle}

The structure of sensor head employed in the present work is illustrated schematically in Fig. 1. The Nafion film deposited on the optical fibre tip forms a Fabry-Perot resonant cavity. The sensing cavity consists of two partially reflective interfaces separated by a distance $L$, which is the thickness of the film. Thus, the reflection at the interfaces of the cavity forms an interference signal at the output. The mirror surfaces are the interfaces between the fibre core-Nafion film and the Nafion film-external environment whose reflectivity depends on the difference of refractive index between each optical medium. As the reflectivity of the interfaces is low, the behaviour of the cavity can be approximated to a two-beam interferometer model (Eq. (1)) [33],

$I=I_{1}+I_{2}+2 \sqrt{I_{1} I_{2}} \cos \left(\phi+\varphi_{0}\right)$

where $I$ is the intensity of the interference signal; $I_{1}$ and $I_{2}$ are the intensities reflected at each interface; $\varphi_{0}$ is the initial phase and $\phi$ the relative phase of the interferometer, given by the expression,

$\phi=\frac{4 \pi n_{2} L}{\lambda}$

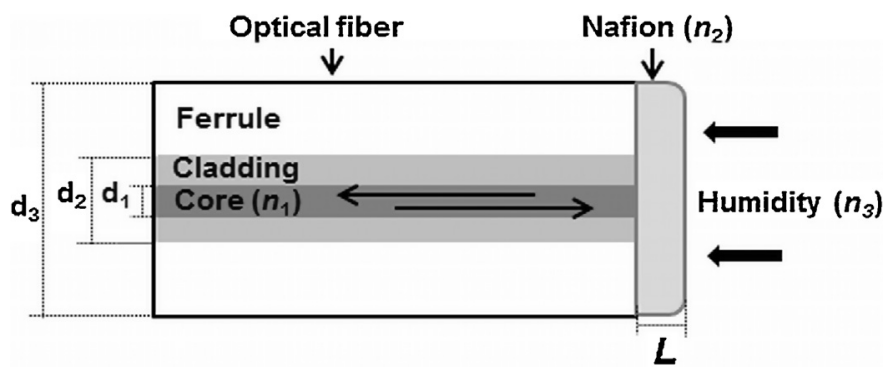

Fig. 1. Schematic showing the structure of the fibre sensor probe $\left(d_{1}=9 \mu \mathrm{m}\right.$. $d_{2}=125 \mu \mathrm{m}$ and $\left.d_{3}=2 \mathrm{~mm}\right)$. where $n_{2}$ is the refractive index of the medium filling the cavity and $\lambda$ is the wavelength of the light source. The phase difference between both interfering beams is dependent upon the thickness, $L$, and the refractive index of the cavity. The periodicity of the fringes of the channelled spectrum can be expressed as,

$\Delta \lambda=\frac{\lambda^{2}}{2 n_{2} L}$

Changes in the cavity due to $\mathrm{RH}$ cause a phase shift in the interference signal that can be retrieved by tracking the wavelength shift of the channelled spectrum using a spectrum analyser. Alternatively, using a readout interferometer and well established demodulation techniques the wavelength shift can be converted into the phase of an electronic carrier signal that can be tracked with higher accuracy [34].

Exposing the cavity to a humid environment changes its thickness and its refractive index, causing a phase change and thereby a wavelength shift on the interference fringes. Briefly, the refractive index reduction caused by the swelling of the Nafion film can be explained through cluster network model [35], which is currently the most acceptable. In this model, the Nafion forms ionic clusters (porous), about spherical, with inverted micellar structure. In its dried state, an average cluster has a radius of about $1.8 \mathrm{~nm}$ and it contains about $26 \mathrm{SO}_{3}{ }^{-}$groups distributed on the inner pore surface. In the swollen state, the diameter increases to about $4 \mathrm{~nm}$ and the number of fixed $\mathrm{SO}_{3}{ }^{-}$groups goes up to $\sim 70$. Under these conditions, each pore is filled with about 1000 water molecules [36]. Moreover, in this case of hydration, its refractive index, which is $n_{\mathrm{D}}=1.38$ when dried, reduces because the refractive index of water is 1.33 .

\section{Experimental}

\subsection{Reagents and solutions}

A 5 wt.\% Nafion solution (in low alcohols and 45\% water) was purchased from Aldrich and employed as received.

\subsection{Sensor probe design}

The sensor probe was assembled by depositing a drop of Nafion solution on the tip of a standard SMF28 single mode fibre (core refractive index of 1.46) to cast a thin hydrophilic coating. Aliquots of $6 \mu \mathrm{L}$ and $9 \mu \mathrm{L}$ were manually deposited on the fibre tip with a micropipette and dried for $24 \mathrm{~h}$ at room temperature. Films with thicknesses of $35 \mu \mathrm{m}$ and $76 \mu \mathrm{m}$ were respectively produced, estimated in the region of the fibre core by means of a digital thickness gage Mitutoyo, ID-C112BS. Fig. 2 shows a picture of the sensor head; it can be seen on the tip of the fibre a transparent film, obtained by deposition of $3 \mu \mathrm{L}$ of Nafion, with the shape of a spherical cap. The repeatability in the film preparation is ca. of $10 \%$; however, this

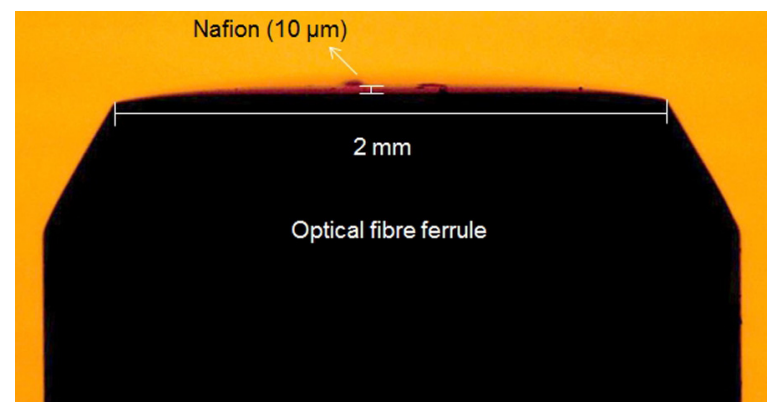

Fig. 2. Picture of the sensor head, showing the Nafion film in the shape of a spherical cap. 


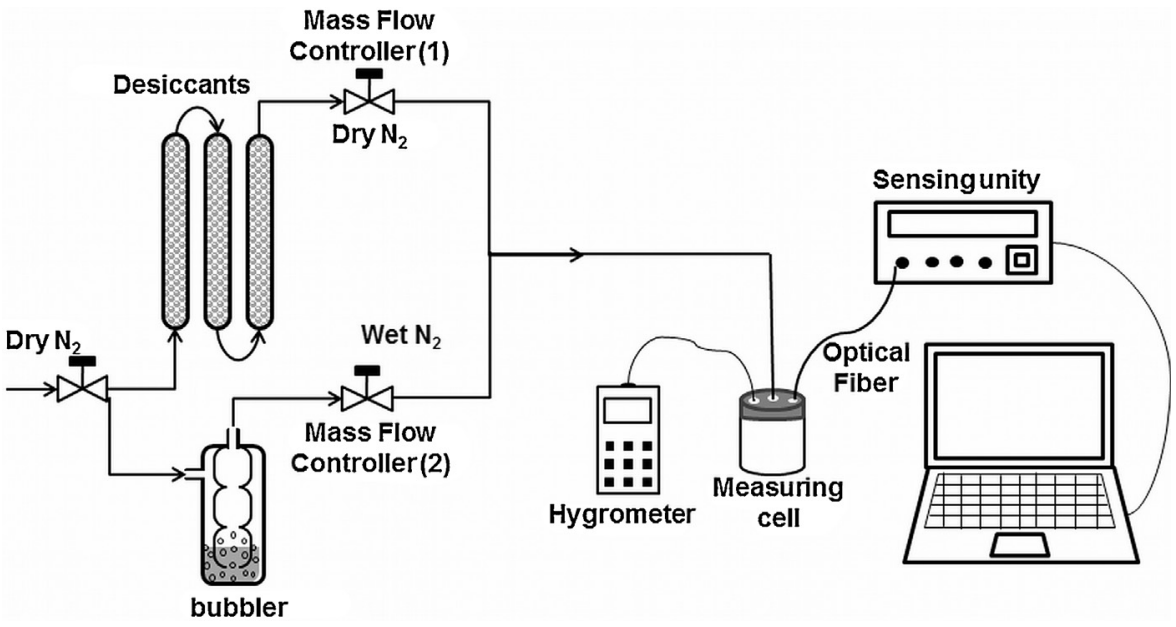

Fig. 3. Schematic diagram of the experimental apparatus used for the sensor characterisation.

fact does not imply a real difficulty, as every new sensor needs to be calibrated before use.

\subsection{Apparatus}

Fig. 3 depicts the experimental setup for humidity measurements. The sensor probe was placed into a measuring cell $(60 \mathrm{~mL})$, whose humidity was controlled by a gas blender, constituted by two mass flow controllers (Aalborg SDPROC TD $9704 \mathrm{M}$ ) with flow rates ranging from 0 to $1000 \mathrm{~mL} \mathrm{~min}^{-1}$. Dry air (<1.0\%RH) was obtained by passing an air flow through desiccant columns of molecular sieve $3 \mathrm{~A}$ (Aldrich), while wet air $(>99 \% \mathrm{RH})$ was obtained by bubbling it into bubbling flasks containing distilled water. Proper mixing of both dry and wet air allowed varying the humidity from 22 to $80 \%$. A hygrometer (Digi-sense humidity data logger model $n^{\circ}$ 37003-02) was also inserted into the cell, in order to provide the relative humidity $(\mathrm{RH})$ inside the chamber, allowing the calibration and evaluation of the sensor. The measurements were made at $(25 \pm 1)^{\circ} \mathrm{C}$. For interference studies, a third mass flow controller (ranging from 0 to $100 \mathrm{~mL} \mathrm{~min}^{-1}$ ) was adapted in the manifold; $6.0 \mathrm{~mol} \mathrm{~L}^{-1} \mathrm{HCl}$ and $0.010 \mathrm{~mol} \mathrm{~L}^{-1}$ ammonia solutions were employed to generate acidic and basic gases, at a concentration of 320 and $400 \mathrm{ppm}_{\mathrm{v}}$, respectively.

Reflectance spectra were obtained with a commercial sensing unit FibreSensing model FS2200SA, working in the 1500-1600 nm range, with $1 \mathrm{pm}$ spectral resolution. The spectra were always acquired after stabilisation of the relative humidity inside the measuring cell.

\section{Results and discussions}

\subsection{Reflection spectra and calibration of relative humidity}

The sensor was studied in the humidity range from 22 to $80 \%$ by using Nafion films with thicknesses of 35 and $76 \mu \mathrm{m}$. Fig. 4 illustrates the interference fringes measured in both extremes of $\mathrm{RH}(22$ and $80 \%$ ) for the two films studied. It was verified that for both cases, the fringes presented a sinusoidal interference pattern as expected.
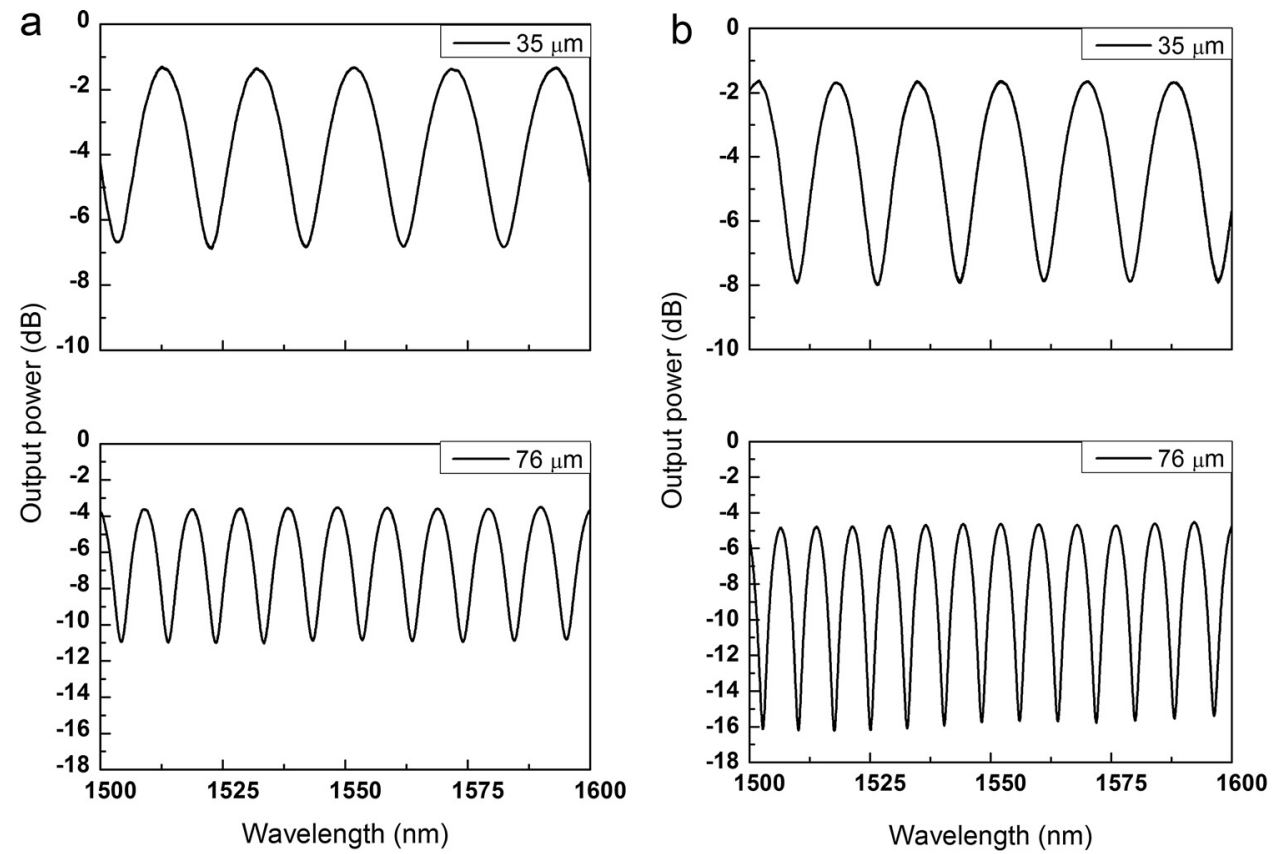

Fig. 4. Reflection spectra measured at the RH of $22 \%$ (a) and $80 \%$ (b) using Nafion film thicknesses of $35 \mu \mathrm{m}$ and $76 \mu \mathrm{m}$. 
Table 1

Variation of the fringe period $\Delta \lambda$ with relative humidity and thickness of Nafion film.

\begin{tabular}{llc}
\hline Film thickness & $\Delta \lambda($ RH 22\%) & $\Delta \lambda($ RH 80\%) \\
\hline $35 \mu \mathrm{m}$ & $19.96 \mathrm{~nm}$ & $17.49 \mathrm{~nm}$ \\
$76 \mu \mathrm{m}$ & $10.09 \mathrm{~nm}$ & $7.80 \mathrm{~nm}$ \\
\hline
\end{tabular}
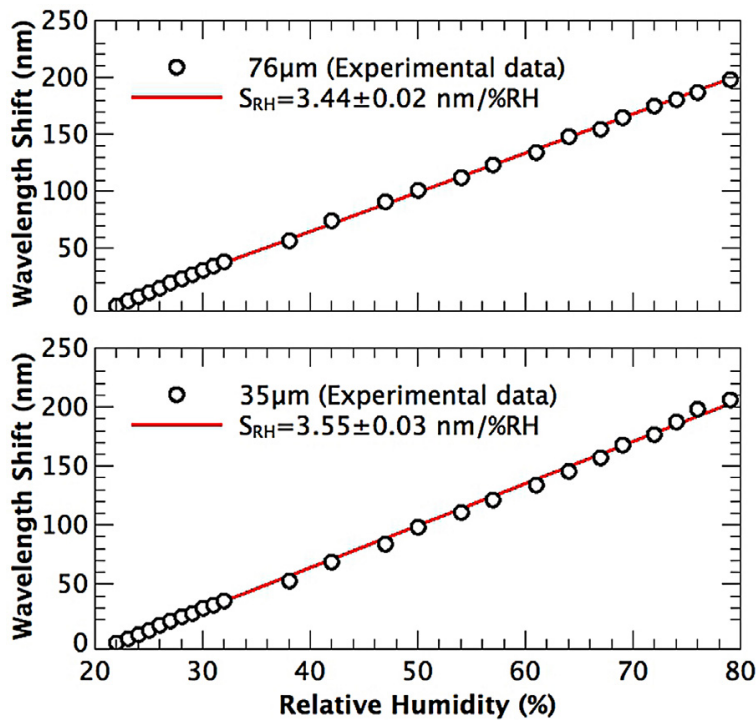

Fig. 5. Analytical curves for the proposed sensor obtained with thicknesses of $35 \mu \mathrm{m}$ and $76 \mu \mathrm{m}$.

The results also reveal that, as foreseeable, that a thicker cavity has a denser interference pattern (i.e. smaller separation between adjacent fringes), which defines the fringe period $(\Delta \lambda)$, as summarised in Table 1.

Fig. 5 shows the analytical curves for relative humidity obtained for the both films studied. The fitting curves display proportional relationship between the wavelength shifts and humidity content, indicating that sensors present linear responses over the relative humidity range studied ( $r^{2}=0.999$ for all curves). Experimental results have also demonstrated that by increasing the $\mathrm{RH}$, the fringes shift towards longer wavelength and $\Delta \lambda$ are reduced (Table 1). The wavelength shift of the fringes and the decrement of $\Delta \lambda$ is consequence of the variation in the refractive index and in the film thickness which are affected by the relative humidity (as it can be inferred by Eq. (3)). The absorption of water by Nafion causes its swelling, which means an increase of the volume (and consequent increase of the geometrical path length for the radiation) and a decrease of the refractive index. Thus, concluding by the experimental results, the swelling of the sensitive membrane is the predominant factor because the combination of the two factors results in an effective increase of the optical path length for the radiation.

Additionally, it can be verified from Fig. 5 that the sensor sensitivity in terms of resulting wavelength shift per \%/RH (i.e. $\mathrm{nm} / \% \mathrm{RH}$ ) with the gradual increase of the film thickness remains practically the same (around $3.5 \mathrm{~nm} / \% \mathrm{RH}$ ), as shown in Table 2. However, it should be considered that the same wavelength shift is being registered for fringe patterns with different periodicity. This way,

Table 2

Effect of the Nafion film thickness on the sensitivity of the sensor estimated in the $22-80 \% \mathrm{RH}$ range.

\begin{tabular}{lll}
\hline & $35 \mu \mathrm{m}$ & $76 \mu \mathrm{m}$ \\
\hline $\mathrm{S}(\mathrm{nm} / \%)$ & $3.56 \pm 0.03$ & $3.44 \pm 0.01$ \\
\hline
\end{tabular}

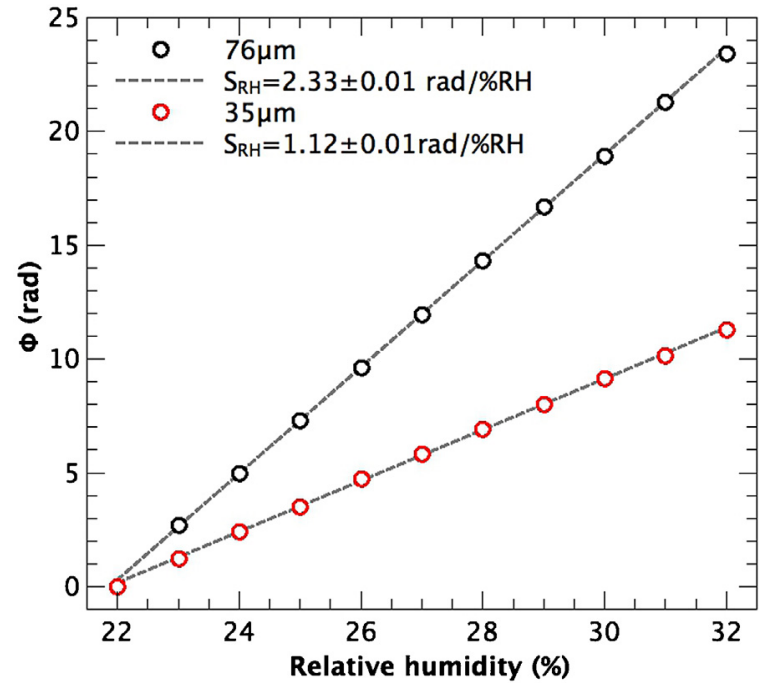

Fig. 6. Estimated phase response as a function of the relative humidity for both sensing cavities in the $22-32 \% \mathrm{RH}$ range. The phase was estimated by normalising the wavelength shift by the period and multiplied by a factor of $2 \pi$.

the sensor sensitivity is better appreciated by considering the corresponding phase shift, since this parameter is intrinsically normalised to the fringe period. Considering Eq. (2) and assuming that a shift with the magnitude of a single period corresponds to a $2 \pi$ phase change, the sensor phase response can easily be derived.

Fig. 6 shows the output graph with the estimated phase response as a function of the relative humidity, for the $22-32 \%$ range. The curve for the cavity of $35 \mu \mathrm{m}$ shows a sensitivity of approximately $1.12 \mathrm{rad} / \% \mathrm{RH}$, while the $76 \mu \mathrm{m}$ cavity shows the highest sensitivity (2.33 rad/\%RH), thus confirming that following Eq. (2), the sensor sensitivity is directly proportional to the cavity length $L$. In a practical system, in order to retrieve the phase information with very high resolution, the fibre probe can be coupled to a serrodyne modulated readout interferometer enabling pseudo-heterodyne interrogation [34,37]. Typically, such system easily attains mrad resolution in phase which, in an ideal situation, would allow to assess the humidity level with $10^{-4} \% \mathrm{RH}$ resolution.

\subsection{Stability and interference studies}

The sensor stability was evaluated using a Nafion film with thickness of $35 \mu \mathrm{m}$ and $\mathrm{RH}$ of $22 \%, 40 \%, 60 \%$ and $80 \%$. For each $\mathrm{RH}$ value, 15 reflection spectra were stored every 9 min during a period of 135 min (Fig. 7).

The stability of the sensor was evaluated by measuring the repeatability (expressed as the standard deviation at a maximum or at a minimum of the fringe) of the spectra obtained as a function of time. As can be seen in Table 3, the sensor showed good stability to humidity at a $22 \% \mathrm{RH}$, with a standard deviation of $0.2 \mathrm{~nm}$. As the relative humidity is increased, the stability of the sensor is decreased, reaching a standard deviation of $2.2 \mathrm{~nm}$ for $80 \% \mathrm{RH}$, which corresponds to a standard deviation of $0.62 \%$ in the relative humidity.

Table 3

Stability of the sensor with a Nafion film thickness of $35 \mu \mathrm{m}$, expressed as the standard deviation in the wavelength measurement.

\begin{tabular}{lll}
\hline $\mathrm{RH}(\%)$ & $\mathrm{SD}(\mathrm{nm})$ & \%RH \\
\hline 22 & 0.2 & 0.06 \\
40 & 0.8 & 0.22 \\
60 & 1.2 & 0.34 \\
80 & 2.2 & 0.62 \\
\hline
\end{tabular}



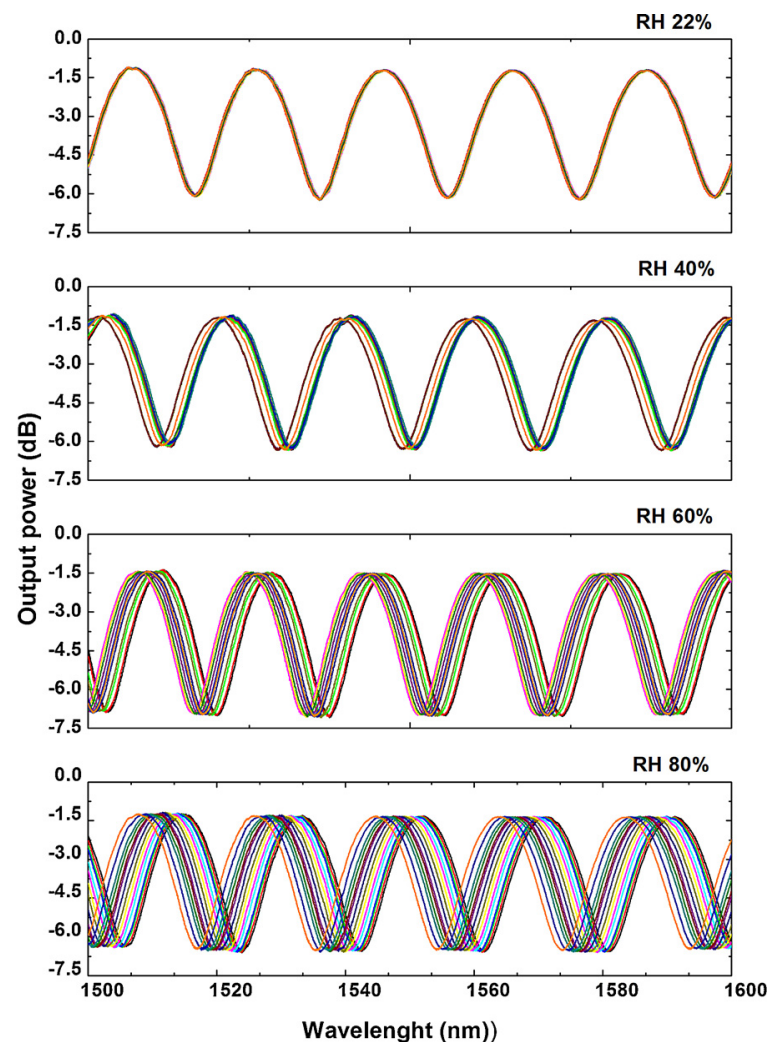

Fig. 7. Stability of the proposed sensor with a film thickness of $35 \mu \mathrm{m}$. For each $\mathrm{RH}$, 15 reflection spectra were stored every 9 min during a period of $135 \mathrm{~min}$.

To complement this study, the long-term sensor stability was also analysed. After 50 days of initial experiments, the sensing phase with thickness of $35 \mu \mathrm{m}$ was subjected to an experiment similar to the initial condition. As can be seen in Fig. 8, both curves show good agreement, demonstrating that the sensor response to the humidity remained almost unchanged, which confirms its durability and good performance.

To evaluate the effect of acidic and basic gases on the sensor response, $\mathrm{HCl}$ and $\mathrm{NH}_{3}$ were employed as models. No interference of $\mathrm{HCl}$ was observed at concentration of $320 \mathrm{ppm}_{\mathrm{v}}$, while ammonia interfered at concentration of $400 \mathrm{ppm}_{\mathrm{v}}$. This interference can be explained based on the fact that ammonium ion can form an ionic pair with the sulphonate group of Nafion, changing its characteristics, such as refractive index and thickness.

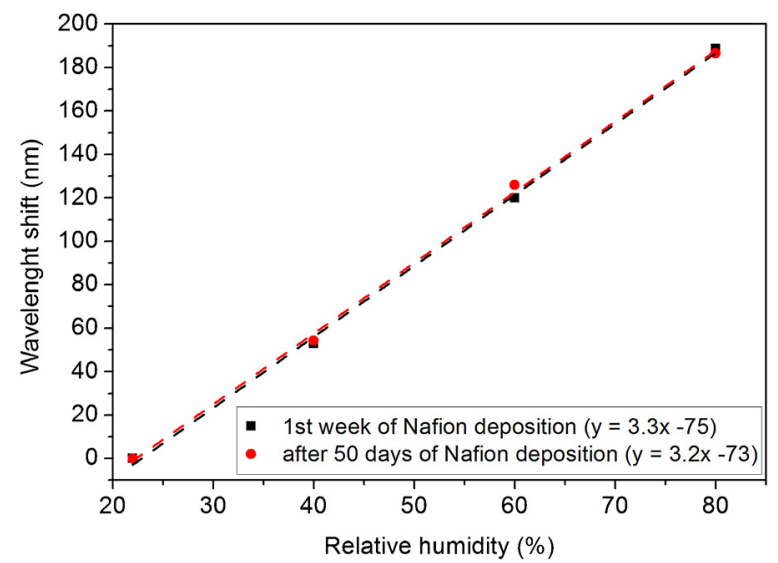

Fig. 8. Sensor responses to humidity (film thickness of $35 \mu \mathrm{m}$ ) obtained in two distinct periods with a time interval of 50 days.
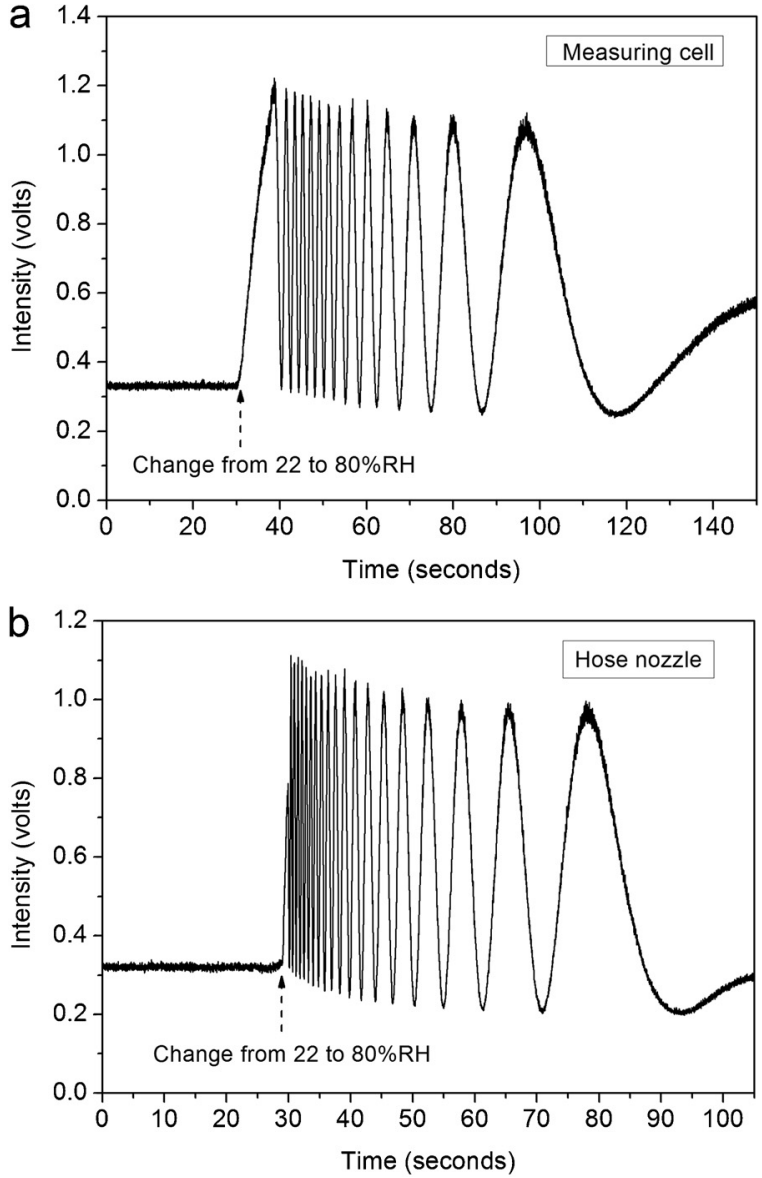

Fig. 9. Response of the sensor (film thickness of $35 \mu \mathrm{m}$ ) as a function of time for RH variation from $22 \%$ to $80 \%$ obtained in the measuring cell (a) and by inserting the fibre tip directly into the hose nozzle (b).

\subsection{Response time}

In order to estimate the response time of the sensor (film thickness of $35 \mu \mathrm{m}$ ) a tunable laser (Model Agilent 8198A) was used and set at $1543 \mathrm{~nm}$. The signal was acquired by photodetector Model 2033 from New Focus. The measurement was performed by exposing the sample to a humidity variation of 58\% (22-80\%) obtaining the signal output in the time domain, as shown in Fig. 9a. A response time of $3.5 \mathrm{~s}$ was estimated for a wavelength shift of half period, which corresponds to a $\mathrm{RH}$ variation of $3 \%$. This response time was estimated from the second oscillation, as the first one encompasses the time necessary for the stabilisation of the mass flow controllers. It can be also inferred from this Figure that it is necessary $25 \mathrm{~s}$ for obtaining a stable signal, referred to the whole variation of $\mathrm{RH}$.

Similarly, the response time of the same sensor was estimated without the measuring cell, inserting the fibre tip with the sensing phase directly into the hose nozzle used for carrying $\mathrm{N}_{2}$. It can be observed in Fig. 9b that for a RH variation of 3\% the time response falls to $242 \mathrm{~ms}$ and it is necessary $11 \mathrm{~s}$ for achieving a steady state signal. These results indicate that the response time depends on the volume of the measuring cell and not of the response time of the sensor itself. In addition, the recovering time was evaluated by varying the humidity from 80 to $22 \%$, being necessary 2 min to reach a steady state signal, fact that demonstrates that desorption of water from the film is slower than the process of absorption. Despite the different in the response times, no hysteresis was observed with the sensor based on the 35- $\mu \mathrm{m}$ Nafion film, as the curve obtained from $80 \%$ to $22 \%$ of relative humidity overlaps that obtained when the humidity is increased (shown in Fig. 5). 


\section{Conclusions}

Nafion was employed as a sensing phase in an optical fibre humidity sensor based on Fabry-Perot interferometry. Due to the high hydrophilic character of the film, the $\mathrm{RH}$ variation affects the amount of water absorbed by the Nafion film, varying its refractive index and thickness. As a consequence, there is wavelength shifts in the reflected light, which is proportional to the relative humidity.

The sensor presents a linear response range from 22 to $80 \% \mathrm{RH}$ and a response time of $242 \mathrm{~ms}$ (3\% RH variation), with a sensitivity estimated as $3.5 \mathrm{~nm} / \% \mathrm{RH}$. Long-term stability was also observed. The phase response was also estimated, and a sensitivity of $2.66 \mathrm{rad} / \% \mathrm{RH}$ was calculated, showing the potential of this sensing probe to measure $\mathrm{RH}$ with high sensitivity and very high accuracy, when coupled with an appropriate readout interferometric system. These results indicate that an optical sensor based on the Fabry-Perot interferometry, which uses Nafion film as resonant cavity, can be employed for monitoring relative humidity in air.

\section{Acknowledgements}

The authors are thankful to INCTAA (CNPq grant 573894/20086 and FAPESP grant 2008/57808-1), Fotonicom, CePOF, and CAPES for financial support. J.S. Santos would like to acknowledge the scholarship from FAPESP (grant 11/00322-2).

\section{References}

[1] R. Demir, Electrical characterization of CdS nanoparticles for humidity sensing applications, Ind. Eng. Chem. Res. 51 (2012) 3309-3313.

[2] R.K. Nahar, On the origin of the humidity sensitive electrical properties of porous aluminium oxide, J. Phys. D: Appl. Phys. 17 (1984) 2087-2095.

[3] I. Emmer, R.G. Pritchard, The influence of some gases on the behaviour of impedance and piezoelectric humidity sensors, Int. J. Electron. 76 (1994) $829-835$.

[4] D. Hudoklin, E. Barukcic, J. Drnovsek, Engaging frost formation in a chilledmirror hygrometer, Int. J. Thermophys. 29 (2008) 1598-1605.

[5] J.A. Kalogiros, C.G. Helmis, Fast response humidity measurements with the psychrometric method, J. Appl. Meteorol. 32 (1993) 1499-1507.

[6] M. Lackner, Tunable diode laser absorption spectroscopy (TDLAS) in the process industries - a review, Rev. Chem. Eng. 23 (2007) 65-147

[7] F.J. Arregui, Y. Liu, I.R. Matias, R.O. Claus, Optical fibre humidity sensor using a nano Fabry-Perot cavity formed by the ionic self-assembly method, IEICE Trans. Electron. E83-C (2000) 360-365.

[8] F. Mitschke, Fibre optic sensor for humidity, Opt. Lett. 14 (1989) 967-969.

[9] P. Wang, F. Gu, L. Zhang, L. Tong, Polymer microfibre rings for high-sensitivity optical humidity sensing, Appl. Opt. 50 (2011) G7-G10.

[10] Y. Wu, T. Zhang, Y. Rao, Y. Gong, Miniature interferometric humidity sensors based on silica/polymer microfibre knot resonators, Sens. Actuators B: Chem. 155 (2011) 258-263.

[11] P.J. Rivero, A. Urrutia, J. Goicoechea, F.J. Arregui, Optical fibre humidity sensors based on localized surface plasmon resonance (LSPR) and lossy-mode resonance (LMR) in overlays loaded with silver nanoparticles, Sens. Actuators B: Chem. 173 (2012) 244-249.

[12] A. Gaston, I. Lozano, F. Perez, F. Auza, J. Sevilla, Evanescent wave optical fibre sensing (temperature, relative humidity, and pH sensors), IEEE Sens. J. 3 (2003) 806-811.

[13] S.K. Khijwania, K.L. Srinivasan, J.P. Singh, An evanescent-wave optical fibre relative humidity sensor with enhanced sensitivity, Sens. Actuators B: Chem. 104 (2005) 217-222

[14] T.E. Brook, M.N. Taib, R. Narayanaswamy, Extending the range of a fibre optic relative humidity sensor, Sens. Actuators B: Chem. 38-39 (1997) 272-276.

[15] P.C.A. Jerônimo, A.N. Araújo, M. Conceição, B.S.M. Montenegro, Optical sensors and biosensors based on sol-gel films, Talanta 72 (2007) 13-27.

[16] B.H. Lee, Y.H. Kim, K.S. Park, J.B. Eom, M.J. Kim, B.S. Rho, H.Y. Choi, Interferometric fibre optic sensors, Sensors 12 (2012) 2467-2486.

[17] L.H. Chen, C.C. Chan, T. Li, M. Shaillender, B. Neu, P. Balamurali, R. Menon, P. Zu, X.M. Ang, W.C. Wong, C.L. Poh, K.C. Leong, Chitosan coated polarization maintaining fibre based Sagnac interferometer for relative humidity measurement, IEEE J. Sel. Top. Quantum Electron. 18 (2012) 1597-1604.

[18] M. Shao, X. Qiao, H. Fu, N. Zhao, Q. Liu, H. Gao, An in fibre Mach-Zehnder interferometer based on arc-induced tapers for high sensitivity humidity sensing, IEEE Sens. J. 13 (2013) 2026-2031

[19] X. Yu, P. Childs, M. Zhang, Y. Liao, J. Ju, W. Jin, Relative humidity sensor based on cascaded long-period gratings with hydrogel coatings and Fourier demodulation, IEEE Photonics Technol. Lett. 21 (2009) 1828-1830.
[20] J. Yao, T. Zhu, D. Duan, M. Deng, Nanocomposite polyacrylamide based open cavity fibre Fabry-Perot humidity sensor, Appl. Opt. 51 (2012) 7643-7647.

[21] L.H. Chen, T. Li, C.C. Chan, R. Menon, P. Balamurali, M. Shaillender, B. Neu, X.M. Ang, P. Zu, W.C. Wong, K.C. Leong, Chitosan based fibre optic Fabry-Perot humidity sensor, Sens. Actuators B: Chem. 169 (2012) 167-172.

[22] T. Li, X. Dong, C.C. Chan, K. Ni, S. Zhang, P.P. Shum, Humidity sensor with a PVA coated photonic crystal fiber interferometer, IEEE Sens. J. 13 (2013) 2214-2216.

[23] J. Mathew, Y. Semenova, G. Farrell, Relative humidity sensor based on an agarose infiltrated photonic crystal fiber interferometer, IEEE J. Sel. Top. Quantum Electron. 18 (2012) 1553-1559.

[24] S. Acikgoz, B. Bilen, M.M. Demir, Y.Z. Menceloglu, Y. Skarlatos, G. Aktas, M.N Inci, Use of polyethylene glycol coatings for optical fibre humidity sensing, Opt. Rev. 15 (2008) 84-90.

[25] G. Sasikumar, J.W. Ihm, H. Ryu, Optimum Nafion content in PEM fuel cell electrodes, Electrochim. Acta 50 (2004) 601-605.

[26] A. Omosebi, R.S. Besser, Electron beam assisted patterning and dry etching of Nafion membranes, J. Electrochem. Soc. 158 (2011) D603-D610.

[27] I.M. Raimundo Jr., R. Narayanaswamy, Evaluation of Nafion-crystal violet films for the construction of an optical relative humidity sensor, Analyst 124 (1999) 1623-1627.

[28] H. Dacres, R. Narayanaswamy, Highly sensitive optical humidity probe, Talanta 69 (2006) 631-636.

[29] X.L. Jin, W. Li, D. Sun, Z. Zhuang, X. Wang, Fabrication of relative humidity optical fibre sensor based on Nafion-crystal violet sensing film, Spectrosc. Spect. Anal. 25 (2005) 1328-1331.

[30] I.M. RaimundoJr, R. Narayanaswamy, Simultaneous determination of relative humidity and ammonia in air employing an optical fibre sensor and artificial neural network, Sens. Actuators B: Chem. 74 (2001) 60-68.

[31] Y. Sadaoka, M. Matsuguchi, Y. Sakai, Optical fibre humidity sensor using nafion R-tri-phenylcarbinol composite, J. Electrochem. Soc. 138 (1991) 614-615.

[32] S.J. Glenn, B.M. Cullum, R.B. Nair, D.A. Nivens, C.J. Murphy, S.M. Angel, Lifetime-based fiber-optic water sensor using a luminescent complex in a lithium-treated Nafion ${ }^{\mathrm{TM}}$ membrane by, Anal. Chim. Acta 488 (2001) 1-8.

[33] J.L. Santos, A.P. Leite, D.A. Jackson, Optical fibre sensing with a low-finesse Fabry-Perot cavity, Appl. Opt. 31 (1992) 7361-7366.

[34] Y. Rao, D. Jackson, Recent progress in fibre optic low-coherence interferometry, Meas. Sci. Technol. 7 (1996) 981-999.

[35] T.D. Gierke, W.Y. Hsu, The cluster-network model of ion clustering in perfluorosulfonated membranes, in: Perfluorinated Ionomer Membranes, ACS Symposium Series, Chapter 13, vol. 180, 1982, pp. 283-307.

[36] B. Smitha, S. Sridhar, A.A. Khan, Solid polymer electrolyte membranes for fuel cell applications - a review, J. Membr. Sci. 259 (2005) 10-26.

[37] C. Gouveia, M. Zibaii, H. Latifi, M.J. Marques, J.M. Baptista, P.A.S. Jorge, Temperature independent refractive index measurement using white light interferometry, in: Proc. SPIE 8421, OFS2012 22nd International Conference on Optical Fibre Sensors, 842160, 2012.

\section{Biographies}

Juliana S. Santos received the BSc degree in Mathematics from Federal University of São Carlos (São Carlos, Brazil), in 2001. In 2003, she joined the Laboratory of Integrated Quartz Cycle (LIQC) in Faculty of Mechanical Engineering from State University of Campinas - UNICAMP (Campinas, Brazil) where she received her MS and $\mathrm{PhD}$ degrees. She has been working in the Group of Instrumentation and Automation in Analytical Chemistry (GIA) in Institute of Chemistry from UNICAMP on the humidity optical fibre sensors.

Ivo M. Raimundo Jr. is associate professor in the Department of Analytical Chemistry of Institute of Chemistry at UNICAMP (Brazil). He received his PhD in Sciences (1995) from UNICAMP and he was post-doctorate fellow (July 1998 to December 1999) at UMIST (Manchester, England). He is member of the Group for Instrumentation and Automation in Analytical Chemistry (UNICAMP) since 1990 and is currently interested in luminescent and near infrared optical sensors for environmental monitoring.

Cristiano M.B. Cordeiro is Assistant Professor in the Institute of Physics of the State University of Campinas (UNICAMP), Brazil, with PhD (2003) in the same University, and postdoc from University of Bath, England. He is head of the Specialty Optica Fibre \& Photonics Materials Laboratory (LaFE) at UNICAMP whose main research areas are the development and application of new types of optical fibres (such as photonic crystal fibres and micro/nanofibres) made of silica, soft glasses or polymers and how to extend its functionalities using new geometries, materials and postprocessing techniques.

Claudecir R. Biazoli graduated in Physics from the University of Campinas (UNICAMP) in 2008. He joined the Specialty Optical Fibre \& Photonics Materials Laboratory (LaFE) at UNICAMP in 2008, conducting researchers in the area of micro and nano fibres for optical sensing application.

Carlos J. Gouveia graduated with a Degree in electronics and telecommunication engineering, in 2007, and the MSc degree in telecommunications and networks, in 2008, from the University of Madeira, Portugal. Currently, he is a PhD student in University of Madeira, working in the Optoelectronics Group of INESC Porto. His research interests are on optical fibre sensors and interrogation systems for chemical and biochemical applications. 
Pedro A.S. Jorge graduated in applied physics (optics and lasers) from the University of Minho in 1996. He received his MSc in optoelectronics and lasers from the physics department of the University of Porto in 2000. In 2006, he concluded his PhD programme at Porto University in collaboration with the department of physics and optical sciences at the University of Charlotte, North Carolina, USA. He is currently a Senior researcher at INESC-Porto where he leads the Biochemical Sensors team exploring the potential of optical fibre and integrated optics technologies in environmental and medical applications coordinating several projects in the area. $\mathrm{He}$ has more than 150 publications in the fields of sensors in national and international conferences and peer reviewed journals, is author of 2 book chapters and also holds one patent. 